\title{
Métodos semiempíricos para la evaluación rápida de orbitales frontera en la clasificación de agonistas y antagonistas de la subunidad NR1 de los receptores iGluR-NMDA
}

\author{
Juvenal Yosa-Reyes ${ }^{1}$, Diana Carolina Clavijo-Buriticá ${ }^{4}$, Carlos Manuel Estévez-Bretón Riveros ${ }^{2}$, \\ Orlando Emilio Acevedo ${ }^{3}$ \\ ${ }^{1}$ Department of Chemistry, Basel University, Klingelbergstrasse $80-4056$, Basel, Switzerland \\ ${ }^{2}$ Gerencia de Proyectos. Futura Networks, SA. Carrera 7 No. 73-00. Bogotá D.C., Colombia. \\ ${ }^{3}$ Departamento de Física, Pontificia Universidad Javeriana. Carrera 7 No. 40-62. Bogotá D.C., Colombia. \\ ${ }^{4}$ Fundação Oswaldo Cruz (FIOCRUZ) Rio de Janeiro, Brasil. \\ *juvenal.yosa@unibas.ch
}

Recibido: 09-09-2010; Aceptado: 27-12-2010

\begin{abstract}
Resumen
Los receptores iGluR-NMDA poseen gran interés farmacológico debido a que están implicados en desórdenes neurodegenerativos y neurosiquiátricos incluso participan en procesos como plasticidad sináptica, esencial para la formación de la memoria. La subunidad NR1 de los iGluR-NMDA es fundamental para que este tipo de receptores se activen apropiadamente, de hecho muchos de los fármacos estudiados para los desórdenes anteriormente mencionados, están dirigidos específicamente a la subunidad NR1. Estudios previos han determinado que el orbital molecular de más baja energía (LUMO), puede ser usado como parámetro para estimar la actividad agonista o antagonista en la subunidad NR1. Objetivo. Evaluar el método semiempírico CNDO para el cálculo rápido de la energía LUMO, con la finalidad de crear un modelo sencillo para el diseño in silico de nuevos fármacos. Materiales y métodos. Fueron seleccionadas 168 moléculas entre agonistas y antagonistas de la subunidad NR1. La energía de cada estructura fue optimizada y posteriormente fueron calculadas las energías de los orbitales frontera, el LogP, la energía total, la capacidad de formar puentes de hidrógeno, la energía de unión y el momento dipolar. Resultados. Se demuestra que la energía LUMO es suficiente para discriminar entre moléculas agonistas y antagonistas de esta subunidad y que el método CNDO evalúa estas propiedades de manera rápida y eficiente. Conclusión. El método CNDO permite el cálculo rápido, generando a futuro procedimientos eficaces para la caracterización de fármacos potenciales que actúen sobre este sitio en particular.
\end{abstract}

Palabras clave: iGluR-NMDA, LUMO, agonista, antagonista, CNDO.

\begin{abstract}
Semiempirical methods for the rapid evaluation of frontier orbitals in the classification of agonists and antagonists of the NR1 subunit of the iGluR-NMDA receptors. The ionotropic glutamate receptors activated by N-Methyl-D-Aspartate (iGluR-NMDA) are of great importance in pharmacology since they are involved in neurodegenerative and neuropsychiatric disorders; they even participate in processes such as synaptic plasticity that are essential for memory formation. Subunit NR1 iGluRs-NMDA is of paramount importance for the appropriate activation of this type of receptors; in fact, many of the pharmaceutical products studied for the abovementioned disorders are targeted specifically to the NR1 subunit. Previous studies have shown that the lowest energy unoccupied molecular orbital (LUMO) can be used as a parameter to estimate the agonist and antagonist activity of the NR1subunit. Objective. Evaluate the semiemprical method CNDO for the rapid calculation of the LUMO energy with the aim of preparing a simple model for the in silico design of new pharmacological substances. Materials and methods. 168 molecules with agonist and antagonist activity in the NR1 subunit were selected. Energy of each structure was optimized and then we calculated the energy of the frontier orbital, the LogP, total energy, capacity of forming hydrogen bonds, binding energy, and dipolar moment. Results. We demonstrate that LUMO energy is enough for discriminating agonist and antagonist
\end{abstract}


molecules of the NR1 subunit and that the CNDO method evaluates these properties in a rapid and efficient way. Conclusions. The CNDO method facilitates a rapid calculation, enabling a future development of effective procedures for the characterization of potential pharmacological substances acting on this particular site.

Key words: iGluR-NMDA, LUMO, agonist, antagonist, CNDO.

\begin{abstract}
Resumo
Métodos semi-empíricos de avaliação rápida de orbitais fronteira na classificação dos agonistas e antagonistas da subunidade NR1 dos receptores iGluR- NMDA. Os receptores IGluR-NMDA têm grande interesse farmacológico porque estão envolvidos em desordens neurodegenerativas e neuropsiquiátricas inclusive participam em processos de plasticidade sináptica, essenciais para a formação da memória. A subunidade NR1 dos iGluR-NMDA é fundamental para que este tipo de receptores se ativem de forma adequada, de fato, muitos dos fármacos estudados para os transtornos mencionados acima, são orientados especificamente pela subunidade NR1. Estudos prévios determinaram que o orbital molecular de mais baixa energia (LUMO), pode ser usado como um parâmetro para estimar a atividade agonista ou antagonista na subunidade NR1. Objetivo. Avaliar o método semi-empírico CNDO para o cálculo rápido da energia LUMO, a fim de criar um modelo simples para o desenho in silicio de novas drogas. Materiais e métodos. Foram selecionadas 168 moléculas entre agonistas e antagonistas da subunidade NR1. A energia de cada estrutura foi otimizada e, em seguida, foram calculadas as energias de orbitais fronteira, o $\operatorname{LogP}$, a energia total, a capacidade de formar pontes de hidrogênio, a energia de ligação e o momento dipolar. Resultados. Foi demonstrado que a energia LUMO é suficiente para discriminar entre moléculas agonistas e antagonistas desta subunidade e que o método CNDO avalia essas propriedades de forma rápida e eficiente. Conclusão. O método CNDO permite o cálculo rápido, gerando a futuro procedimentos eficazes para a caracterização de potenciais medicamentos que agem neste sitio em particular.
\end{abstract}

Palavras-chave: iGluR-NMDA, LUMO, agonista, antagonista, CNDO.

\section{Introducción}

Los miembros de la familia de receptores de glutamato (GluRs), intervienen principalmente en la neurotransmisión excitatoria en el cerebro de los mamíferos. Los GluRs básicamente comprenden dos familias: (i) los receptores metabotrópicos (mGluRs), que no están asociados a canales iónicos, y (ii) los receptores ionotrópicos (iGluRs), asociados a canales catiónicos. Los iGluRs se subdividen a su vez en dos tipos, los no-NMDA (iGluR-noNMDA), entre ellos se encuentran los receptores AMPA (a-amino-3hidroxi-5-metil-4-isoxasol propionato) y kainato ((2S,3S,4S)-3-(carboxymethyl)-4-prop-1-en-2-ilpirrolidin2-carboxilato). El segundo tipo está constituido por los receptores activados por N-Metil-D-Aspartato (iGluRNMDA).

Los iGluR-NMDA son de gran interés farmacológico, debido a que están implicados en varios desórdenes neurodegenerativos, entre los que se encuentran Alzheimer, Huntington y la enfermedad de parkinson, de igual manera están involucrados en enfermedades de tipo neurosiquiátricas como la esquizofrenia y la depresión, también en desórdenes provocados por el consumo de alcohol y sustancias psicotrópicas. Se ha demostrado que los iGluR-NMDA están involucrados en la sensación de dolor y en procesos importantes como la plasticidad sináptica, que es esencial para la formación de la memoria (1). Los iGluR-NMDA son activados por glutamato, siendo su agonista natural; sin embargo, para su funcionamiento como canal, requieren de la presencia de glicina como agonista (2) y de moduladores como diferentes tipos de poliaminas, $\mathrm{Zn}^{2+}$, al igual que del potencial redox del medio circundante. Cuando los iGluR-NMDA son activados, el canal iónico se abre permitiendo la entrada de calcio $\left(\mathrm{Ca}^{+2}\right)$ (3). Este último proceso es regulado por el cambio del potencial electroquímico y por iones de magnesio $\left(\mathrm{Mg}^{2+}\right)$ (4-5). Adicionalmente el canal hace simporte con sodio $\left(\mathrm{Na}^{+}\right)$y antiporte con potasio $\left(\mathrm{K}^{+}\right)$.

Los iGluR-NMDA son un complejo que contiene de 3 a 5 subunidades (6). Las más comúnmente encontradas formando diferentes estequiometrías en este receptor incluyen la NR1 NR2 (A-D) (7) y NR3 (A-B) (8). La subunidad NR1 parece ser clave en la formación del canal, además de contener el sitio de unión a glicina, que a diferencia de los receptores de glicina (sensibles a estricnina), este tipo en particular es excitatorio. Por su parte la subunidad NR2 contiene el sitio de unión a glutamato.

Los iGluR-NMDA poseen varios sitios alostéricos por los cuales es modulado (9-10), entre ellos se encuentran el sitio de unión a poliaminas, el sitio de unión a zinc $\left(\mathrm{Zn}^{+2}\right)$ y el sitio de fosforilación. Además de los mencionados anteriormente, los iGluR-NMDA son modulados por $\mathrm{pH}$ y potenciales redox. Varias sustancias como la fenilciclidina (PCP) y MK-801 pueden bloquear el canal (11-12). 
Este estudio se centra en cómo algunas propiedades electrónicas asociadas a los compuestos que interactúan con el sitio de glicina, están involucradas en la activación de los iGluR-NMDA. Se seleccionaron 168 compuestos con actividad agonista y antagonista sobre la subunidad NR1 (22 agonistas y 146 antagonistas) (13-25). A cada compuesto le fueron evaluadas algunas propiedades de tipo fisicoquímico y electrónico, usando para este último el método hamiltoniano semiempírico CNDO (del inglés Complete Neglect Differentiation Orbital).

\section{Materiales y métodos}

Los cálculos aquí realizados se basan en estudios previos hechos por Yosa et al., en los cuales se afirma que el orbital desocupado de menor energía (LUMO) es un factor crítico en la división entre agonistas y antagonistas para las sustancias que actúan sobre la subunidad NR1 de los receptores iGluR-NMDA. Para este estudio se tomaron las mismas moléculas analizadas por Yosa et al., (13-25), teniendo como objetivo evaluar el método CNDO que permite calcular de manera más rápida que los métodos usados por Yosa et al., de esta manera comparar los resultados aquí obtenidos con los llevados a cabo anteriormente usando el funcional de la densidad. La diferencia entre los estudios previos y los resultados aquí propuestos radica principalmente en que CNDO por ser un método de tipo semiempírico lleva a cabo los cálculos mucho más rápido que usando la teoría del funcional de la densidad, en cuyo caso podría calcular miles incluso millones de moléculas para la rápida evaluación y determinación de sustancias agonistas o antagonistas.

Todas las estructuras fueron modeladas usando el software comercial Molecular Modeling Pro. versión 5.2.4. (26). Cada estructura fue optimizada, los orbitales frontera (HOMO y LUMO), la energía total de cada sistema molecular al igual que los parámetros físicos y fisicoquímicos, como: energía de unión, dipolo momentáneo, aceptor y donador de puentes de hidrógeno y el coeficiente de partición octanol/agua $(\log \mathrm{P})$, fueron obtenidos usando CNDO. Para las pruebas estadísticas se usó el protocolo propuesto por Yosa et al. (26), en el cual la evaluación de la normalidad para cada distribución se realizó por medio de dos pruebas estadísticas: Shapiro-Wilks (27) y Kolmogorov-Smirnov (28). Los test para dos muestras independientes fueron evaluados usando las pruebas de Wald-Wolfowitz, Kolmogorov-Smirnov y la U de Mann-Whitney. Las pruebas de normalidad al igual que las pruebas para dos muestras independientes fueron realizadas usando el paquete estadístico Statistica 6.0.
Los métodos semiempíricos se refieren al tipo de cálculos en mecánica cuántica, que usan parámetros derivados de experimentos para simplificar el proceso de resolver las ecuaciones de onda. El modelo semiempírico utiliza la ecuación de Roothaan-Hall como punto de partida. Las restricciones y demás aproximaciones son luego aplicadas con el fin de reducir el cálculo computacional. El rendimiento de los métodos semiempíricos puede ser evaluado por comparación con geometrías en equilibrio obtenidas experimentalmente, frecuencias vibracionales, energías relativas, dipolos momentáneos, propiedades termodinámicas, etc. para moléculas estables. Los métodos semiempíricos son menos costosos en tiempo de procesamiento, que los métodos $a b$ initio o el funcional de la densidad.

El modelo usado es confinado a moléculas de capa cerrada, en el cual los electrones de valencia se mueven alrededor de un centro el cual incluye los electrones más internos (aproximación "core" o centro). Los orbitales moleculares de la capa de valencia $\left(\psi_{\mathrm{i}}\right)$ son representados por combinaciones lineales de conjuntos de base mínimos de la capa de valencia de los orbitales atómicos $\left(\phi_{\mu}\right)$ :

$$
\psi_{i}=\sum_{\mu=1}^{N} C_{\mu i} \phi_{\mu}
$$

Aquí los orbitales atómicos son orbitales tipo Slater de la forma:

$$
\phi_{\mu}=N e^{-\zeta r} Y_{l}^{m}(\theta, \phi)
$$

Donde $\zeta$ es el exponente del orbital, $\mathrm{Y}_{l}^{m}$ son los esféricos armónicos reales. El exponente representa la carga nuclear efectiva que experimenta un electrón en el orbital atómico. Si tomamos la ecuación de Roothan-Hall:

$$
\sum_{v=1}^{N}\left(F_{\mu v}-\varepsilon_{i} S_{\mu v}\right) C_{v i}=0, \quad \mu=1,2, \ldots, N
$$

Con condiciones de normalización se obtiene:

$$
\sum_{\mu=1}^{N} \sum_{v=1}^{N} C_{\mu i} S_{\mu v} C_{v i}
$$

$\varepsilon_{\mathrm{i}}$ de la ecuación 3, es la energía de un-electrón del orbital molecular $\psi_{\mathrm{i}}, \mathrm{S}_{\mu v}$ son los elementos de la matriz $\mathrm{NxN}$ denominada matriz de solapamiento.

$$
S_{\mu v}=\int \phi_{\mu}(1) \phi_{v}(1) d x_{1} d y_{1} d z_{1}
$$


Donde $\phi_{\mu}$ es el orbital atómico del átomo $\mathrm{A}$ y $\phi_{\mathrm{v}}$ es el orbital atómico del átomo B. $\mathrm{F}_{\mu \nu}$ de la ecuación 3 son los elementos de otra matriz $\mathrm{N}$ x N, denominada matriz Fock:

$$
F_{\mu \nu}=H \mu \nu r e+\sum_{\lambda=1}^{N} \sum_{\sigma=1}^{N} P_{\lambda \sigma}\left[(\mu \nu \mid \lambda \sigma)-\frac{1}{2}(\mu \nu \mid \lambda \sigma)\right]
$$

Donde $\mathrm{H}_{\mu v}$ core es la matriz que representa la energía de un solo electrón en el campo "descubierto" del núcleo. Estos elementos son:

$H_{\mu \nu}^{\text {core }}+(1)=-\frac{1}{2}\left(\frac{\partial^{2}}{\partial x^{2}}+\frac{\partial^{2}}{\partial y^{2}}+\frac{\partial^{2}}{\partial z^{2}}\right)-\sum_{A=1}^{M} \frac{Z_{\mathrm{A}}}{\mathrm{A}_{1 \mathrm{~A}}}$

Aquí $\mathrm{Z}_{\mathrm{A}}$ es el número atómico del átomo $\mathrm{A}$ y la sumatoria se lleva a cabo sobre todos los átomos. El término $\langle\mu \nu \mid \sigma \lambda\rangle$ en [6] son las integrales de repulsión para dos-electrones:

$$
(\mu \nu \mid \lambda \sigma)=\iint \phi_{\mu}(1)\left(\frac{1}{\mathrm{R}_{12}}\right) \phi_{\lambda}(2) \phi_{\sigma}(2) d x_{l} d y_{l} d z_{l}
$$

La ecuación anterior es multiplicada luego por la matriz de densidad de un-electrón $\mathrm{P}_{\lambda \sigma}$ :

$$
P_{\lambda \sigma}=2 \sum_{i=1}^{O C C} C_{\lambda_{i}} C_{\sigma i}
$$

La sumatoria se hace solamente sobre los orbitales ocupados. El factor 2 indica que hay dos electrones ocupando cada orbital molecular, y el asterisco indica una conjugación compleja (requerida en el caso de que el orbital molecular no sea una función real). La energía Total $E^{\text {tot }}$ de la molécula es la suma de la energía electrónica $E_{\text {el }}$ y la energía de repulsión $\mathrm{E}_{\mathrm{AB}}{ }^{\text {core }}$ entre los centros de los átomos A y B:

$$
E_{\text {mol }}^{\text {tot }}=E_{e l}+\sum \sum_{A<B} E_{A B}^{c o r e}
$$

CNDO fue desarrollado por Pople, Segal y Santry (29) y las aproximaciones usadas son las siguientes; la matriz Fock, ecuación [3], no tiene en cuenta la diferencial de solapamiento. Todas las integrales de solapamiento que involucran los diferentes orbitales atómicos, ecuación [5], son ajustadas a 0 . Todas las nubes de carga derivadas de los diferentes orbitales atómicos, $\Phi_{\mu}$, son ignoradas, esto elimina la mayoría de integrales de dos-electrones multicentros de tal manera que:

$$
(\mu \nu \mid \lambda \sigma)=\delta(\mu, v) \delta(\lambda, \sigma)(\mu v \mid \lambda \sigma)
$$

Donde: $\delta(\mu, v)=1$ si $\mu$ es igual a $v$, de lo contrario será 0 .
Todas las integrales dos-centros dos-átomos entre un par de átomos son ajustadas de la siguiente manera:

$$
(\mu \nu \mid \lambda \sigma)=Y_{A B}
$$

Donde: $\mathrm{Y}_{\mathrm{AB}}$ es una función de los átomos A y $\mathrm{B}$ asociada a la distancia intraatómica únicamente. Todas las interacciones centros-electrones para un determinado par de átomos se ajustan de la siguiente forma:

$$
\left(\mu\left|V_{B}\right| v\right)=\delta(\mu, v) V_{A B}
$$

Donde: $\mathrm{V}_{\mathrm{AB}}$ es la energía de interacción de un electrón $\mathrm{A}$ en un orbital de valencia con su centro (núcleo + capa interna) de $\mathrm{B}$. $\mathrm{V}_{\mathrm{AB}}$ es corregido en CNDO incluyendo la desigualdad de la atracción electrón-centro y la repulsión electróncentro.

$$
V_{A B}=Z_{B} Y_{A B}
$$

Donde: $Y_{A B}$ es un promedio de la energía de interacción de un electrón en cualquier orbital atómico de valencia A con otro en un orbital B. Las integrales un-electrón "offdiagonal" o resonancia son ajustadas proporcionalmente a la integral de solapamiento.

$$
H_{\mu v}=\beta_{A B} S_{\mu v}
$$

Donde: $\beta_{\mathrm{AB}}$ es un parámetro el cual depende únicamente de la naturaleza de los átomos A y B. Las interacciones centro-centro en CNDO son entonces:

$$
E_{A B}=Z_{A} Z_{B} / R_{A B}
$$

Donde: $Z_{A}, Z_{B}$ es la carga del núcleo con los electrones de la capa interna colapsados. En CNDO la integral un-electrón un-centro, $\mathrm{U}_{\mu \mu}$ es el promedio de la energía requerida para remover un electrón de un orbital atómico en un átomo completamente ionizado y la energía liberada cuando el átomo ionizado gana un electrón:

$$
U_{\mu \mu}=-\frac{1}{2}\left(I_{\mu}+A_{\mu}\right)-\left(Z_{A}-\frac{1}{2}\right) Y_{A A}
$$

El elemento de la diagonal de la matriz Fock en CNDO es entonces:

$$
\begin{gathered}
F_{\mu \mu}=-\frac{1}{2}\left(1 \mu+A_{\mu}\right)-\left(Z_{A}-\frac{1}{2}\right) Y_{A A}-\sum_{B \neq \mathrm{A}} Z_{B} Y_{A B} \text { un-electrón } \\
+\left(P_{A A} \frac{1}{2}-P_{\mu \mu}\right)=\sum_{B \neq \mathrm{A}} P_{B B} Y_{A B} \quad \text { dos-electrones }
\end{gathered}
$$


Y el término "off-diagonal" se convierte entonces en:

$$
F_{\mu \nu}=\beta_{A B} S_{\mu \nu}-\frac{1}{2} P_{\mu \nu} Y_{A B}
$$

\section{Resultados y discusión}

La forma en que la "off-diagonal" finalmente resulta, ecuación [19], y el resto de aproximaciones de CNDO hacen que el cálculo computacional disminuya significativamente. Los parámetros para los átomos comunes (C, H, O, N, S, etc.) son integrados en el algoritmo de tal manera que todas las moléculas que fueron evaluadas convergen en un mínimo energético.

Una vez cada una de las moléculas fue optimizada se calcularon los parámetros mencionados previamente (ver métodos). Además de las energías de los orbitales frontera y la energía total, para este estudio se calculó también la energía de unión (diferencia de la energía de la molécula en una geometría de equilibrio y la suma de las energías de los átomos constituyentes en la molécula), la capacidad de actuar como donador o aceptor de puentes de hidrógeno (HBD y HBA) y el coeficiente de partición octanol/agua $(\operatorname{LogP})$. Esto con el fin de observar además de la energía LUMO, si existe otra variable que pueda diferenciar entre agonistas y antagonistas.

Las pruebas de Shapiro-Wilk y Kolmogorov-Smirnov fueron empleadas para evaluar si las variables calculadas presentan una distribución normal. La hipótesis nula Ho para cada prueba se define como: los datos se encuentran normalmente distribuidos y la hipótesis alterna Hi se define como: los datos no están normalmente distribuidos. Los resultados para cada variable usando las pruebas de normalidad, se muestran en la Tabla 1. Los valores de $p$ para ambas pruebas se observa que ninguna de las variables usadas muestran una distribución normal (Shapiro-Wilk $\mathrm{p}<0,02$ y Kolmogorov-Smirnov $\mathrm{p}<0,2)$ de tal manera que es rechazada Ho y en consecuencia aceptada Hi. Una gráfica de distribución para la variable de interés se muestra en la Figura 1, claramente se observa que para esta población la distribución no es normal, incluso muestra una tendencia a hacer bimodal.

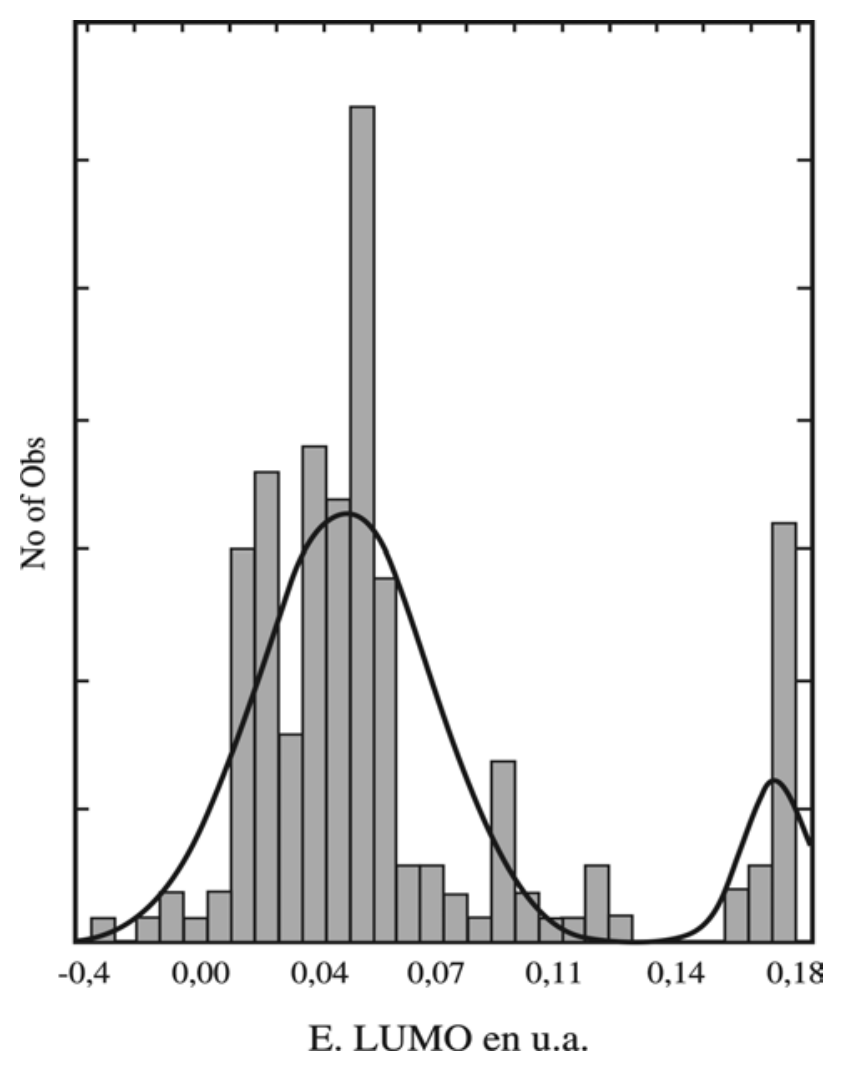

Figura 1. Histograma LUMO (CNDO). Muestra la distribución de la variable LUMO calculada usando CNDO para todas las moléculas empleadas en este estudio, incluyendo agonistas y antagonistas. La energía es expresada en Hartrees.

Tabla 1. Pruebas de normalidad Kolmogorov-Smirnov y Shapiro-Wilks. Resultados obtenidos de cálculos con CNDO

\begin{tabular}{lcccc}
\hline Variable & Ks-d & p & Shapiro-Wilk W & p \\
\hline Energía total & 0,067 & $>0,20$ & 0,982 & 0,02410 \\
Energía de unión & 0,106 & $<0,05$ & 0,961 & 0,00008 \\
HOMO & 0,118 & $<0,050,960$ & 0,00007 & 0,00000 \\
LUMO & 0,249 & $<0,010,806$ & 0,947 & 0,00000 \\
Dipolo momentáneo & 0,096 & $<0,10$ & 0,00453 & 0,00000 \\
HBA & 0,089 & $<0,150,974$ & 0,899 & 0,00003 \\
HBD & 0,170 & $<0,01$ & 0,956 & \\
LogP 0,081 & $<0,20$ & 0,000 & \\
\hline
\end{tabular}


Con el fin de observar la tendencia de cada variable calculada se creó una matriz de dispersión, Figura 2. En esta matriz de dispersión hay varios puntos a destacar, el primero es por ejemplo la correlación existente entre la energía total y la energía de unión, esta correlación no tiene ningún significado físico y tal vez sea debido a que la energía de unión es derivada de la energía de equilibrio de la molécula. Es posible observar también una tendencia lineal de pendiente negativa entre la energía total y $\log P$, esta tendencia es clara para todas las moléculas pero no un parámetro que indique una división entre la actividad agonista o antagonista de las moléculas en estudio. Los gráficos con mayor interés se evidencian entre la energía LUMO y el resto de variables, para este caso en particular se pueden observar dos grupos de moléculas bien diferenciados, al igual que en la distribución en la figura 1. El cluster principal (el de mayor población) muestra una energía menor comparada con el cluster de menor población que muestra una energía significativamente más alta. En el caso de los gráficos que incluyen dipolos momentáneos se observa una leve tendencia a formar dos clusters al igual que LUMO, pero en este caso no están claramente diferenciados como los que muestra la variable LUMO.

\section{Pruebas de independencia de grupos, estadística no paramétrica}

En concordancia con las pruebas de normalidad y de acuerdo con los gráficos de dispersión observados en la figura 2, y aunque ninguna de las variables sigue una distribución gaussiana, sólo dos variables en este caso podrían diferenciar entre la actividad agonista o antagonista de las sustancias aquí evaluadas. Con el fin de establecer cuál de las variables puede de alguna forma diferenciar la actividad, se realizaron
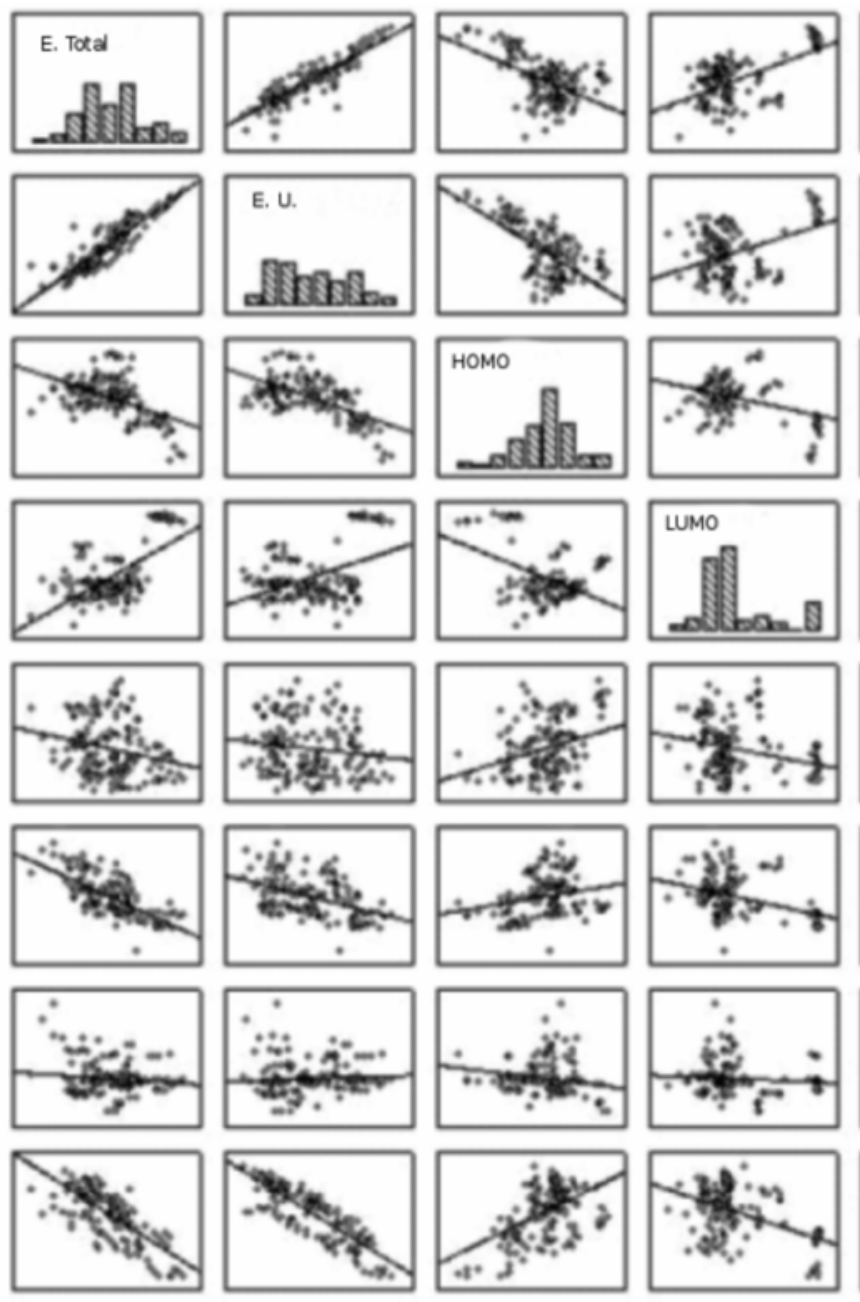
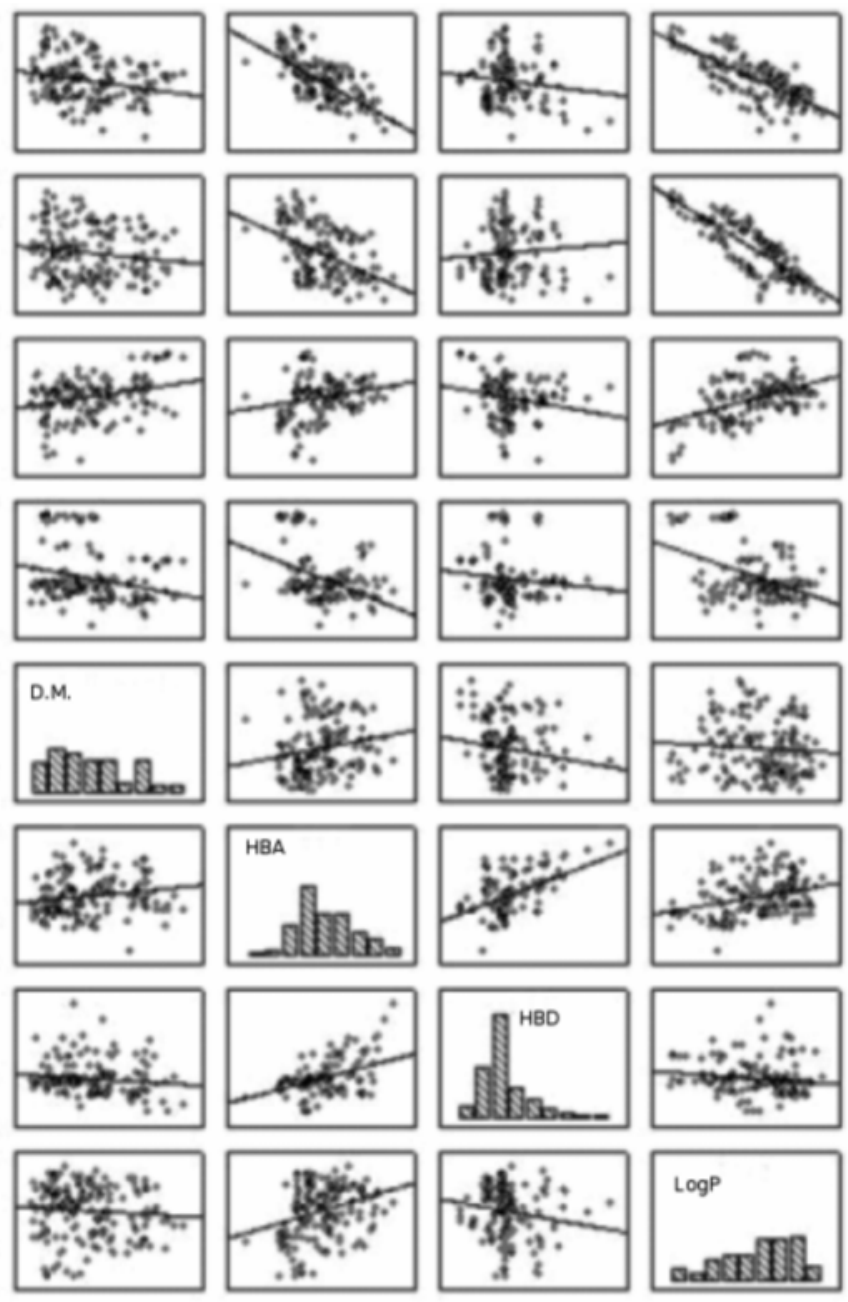

Figura 2. Matriz de dispersión. En la diagonal se presentan los valores de cada una de las variables calculadas usando CNDO. El eje $x$ representa la variable en la correspondiente columna, por su parte el eje $y$ representa la variable en la correspondiente fila. 

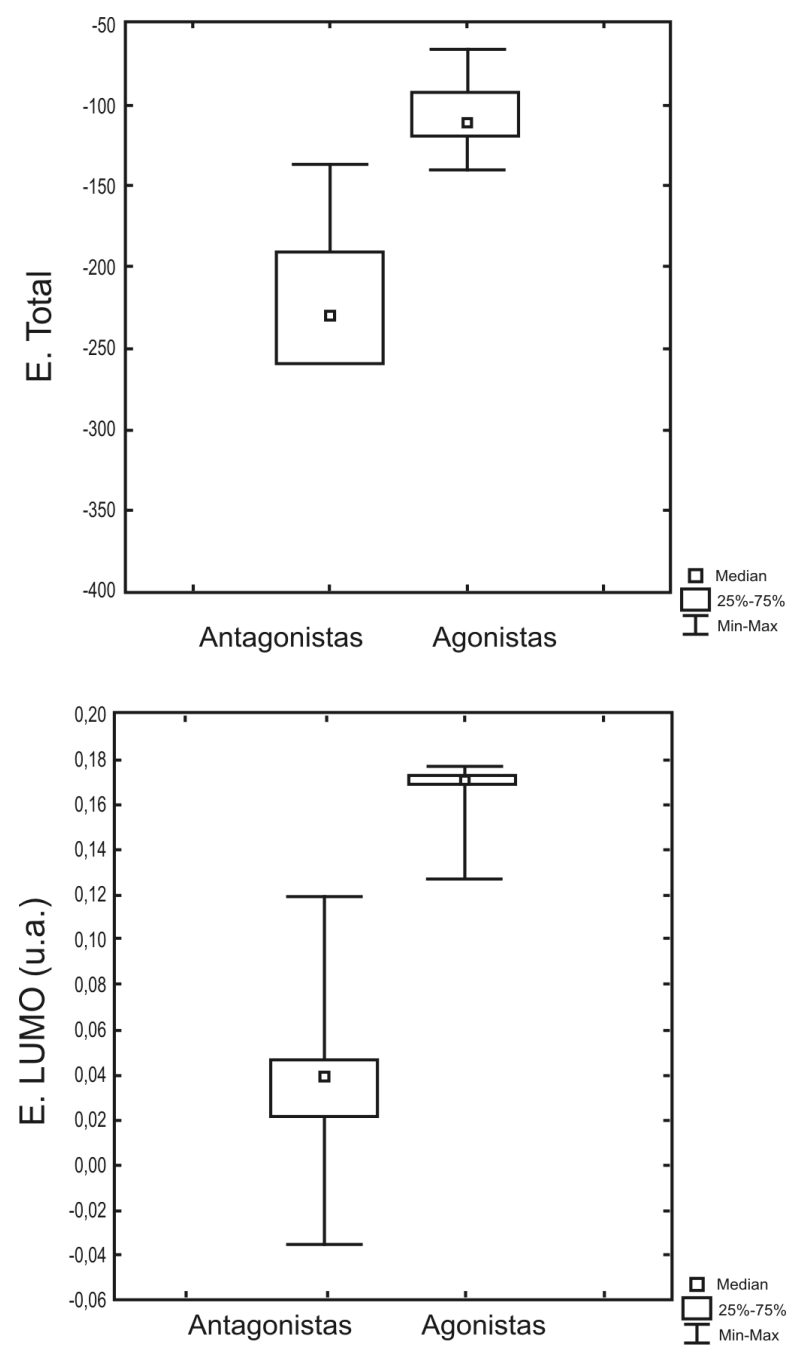

Figura 3. Diagrama de cajas para la variable LUMO. Una fuerte diferencia existente entre los dos grupos, que son clasificados de acuerdo a su actividad agonistas $v s$. antagonistas. La energía es expresada en Hartrees. pruebas estadísticas de independencias de grupos. Los resultados son resumidos en la Tabla 2.

El carácter bimodal o la presencia de dos diferentes poblaciones que coexisten en la misma muestra, son evaluados entonces sabiendo que la hipótesis Ho, es aceptada cuando la probabilidad para diferencias significativas es $>0,05$; luego ambos subgrupos provienen de muestras idénticas por lo que pertenecen a la misma población. Cuando p $<0,05$ se descarta Ho y se acepta Hi (los dos subgrupos pertenecen a poblaciones diferentes). Para todos los casos se obtuvieron resultados en donde era posible observar dos grupos claramente diferenciados, pero en la mayoría de las variables se encontraron datos solapados, esto es, no había una clara diferencia entre actividad agonista o antagonista, únicamente para la variable LUMO se ve esta diferencia marcada, las dos poblaciones observadas se diferencian en su actividad, estos datos soportan los estudios previos realizados por Yosa et al., (26), en donde usando DFT y PM3 se obtuvieron los mismos resultados. Se puede incluso observar esta tendencia a la separación de grupos por actividad en el diagrama de caja que se muestra en la figura 3 para la variable LUMO, donde se evidencia una división entre las dos poblaciones sin solapamientos entre agonistas o antagonistas (sin datos comunes para las dos poblaciones). Al graficar un histograma categórico Figura 4, se muestra una separación de al menos 0,019 au lo que significa que la separación energética para agonistas y antagonistas es alrededor de $12 \mathrm{kcal} / \mathrm{mol}$.

Se construyó un diagrama de dispersión Figura 5, entre la energía total HOMO y LUMO en donde se puede observar los dos grupos claramente diferenciables por actividad en donde la energía LUMO es la variable que es capaz de diferenciar entre las dos poblaciones. De este modo CNDO

Tabla 2. Pruebas para dos poblaciones independientes: Mann_Withney U test, Wald-Wolfowitz Runs y Kolmogorov-Smirnov.

\begin{tabular}{|c|c|c|c|c|c|}
\hline \multirow[b]{2}{*}{ Variable } & \multicolumn{2}{|c|}{ Wald-Wolfowitz } & \multirow{2}{*}{$\frac{\text { Kolmogorov-Smirnov }}{\text { p }}$} & \multicolumn{2}{|c|}{ U Mann-Whitney } \\
\hline & $\mathbf{Z}$ & $\mathbf{p}$ & & $\mathbf{U}$ & $\mathbf{p}$ \\
\hline Energía Enlace -10,24 & $8,0 \mathrm{E}-24$ & $<0,001$ & 66 & $3,4 \mathrm{E}-13$ & \\
\hline HOMO & $-9,54$ & $7,3 \mathrm{E}-21$ & $<0,001$ & 110 & $1,4 \mathrm{E}-12$ \\
\hline LUMO & $-13,02$ & 0,0 & $<0,001$ & 0 & $3,6 \mathrm{E}-14$ \\
\hline Dipolo M. $-2,25$ & $3,8 \mathrm{E}-02$ & $<0,005892$ & $3,7 \mathrm{E}-04$ & & \\
\hline HBA - 8,50 & $8,3 \mathrm{E}-17$ & $<0,001218$ & $4,3 \mathrm{E}-11$ & & \\
\hline HBD & $-3,64$ & $5,3 \mathrm{E}-04>0,10$ & 1639 & $8,4 \mathrm{E}-01$ & \\
\hline $\log \mathrm{P}-7,46$ & $3,2 \mathrm{E}-13$ & $<0,001$ & 293 & $4,0 \mathrm{E}-10$ & \\
\hline
\end{tabular}




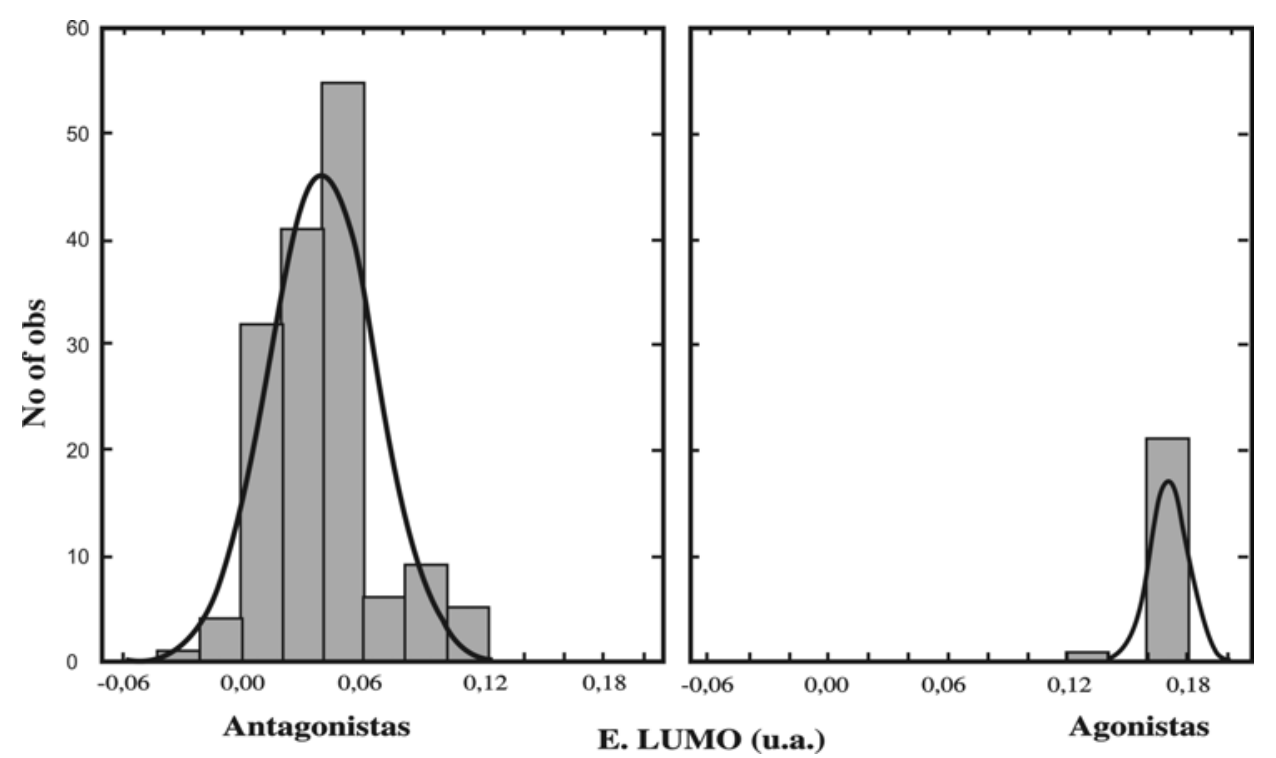

Figura 4. Histograma categorizado. Dos grupos bien diferenciados son observados en función de la variable categórica (actividad agonista o antagonista). La energía es expresada en Hartrees.

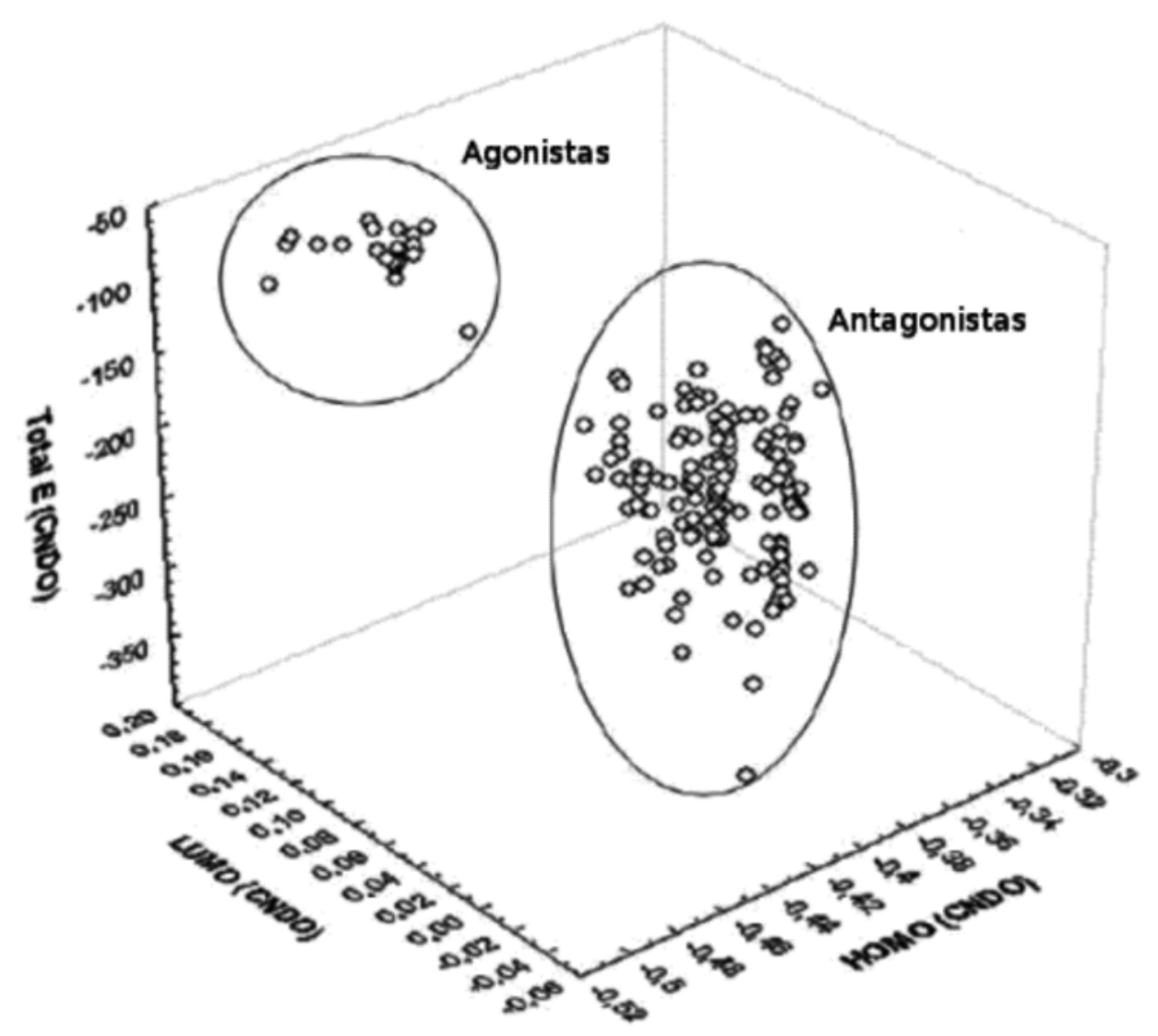

Figura 5. 3D Plot. Energía total, LUMO y HOMO, la energía es expresada en Hartrees. 
se convierte entonces en una herramienta poderosa para ser usada en la rápida clasificación de actividad para la subunidad NR1 de los iGluR-NMDA, incluso podría pensarse en el diseño y programación de un workflow, que integre el cálculo con CNDO y las pruebas estadísticas o incluso acondicionar la utilización de modelos como análisis discriminante o logit-probit, para generar modelos de predicción en donde la evaluación podría llevarse a cabo en tan solo pocos minutos.

\section{Conclusiones}

Simulaciones usando el método semiempírico CNDO creado por Pople, Segal y Santry fueron llevadas a cabo sobre compuestos agonistas y antagonistas de la subunidad NR1 de los receptores iGluR-NMDA. Usando la metodología propuesta por Yosa et al. Se logró corroborar que la energía del orbital molecular de más baja energía (LUMO) puede ser usada como variable de diferenciación entre actividad agonista y antagonista para la renombrada subunidad, se propone entonces de esta manera a CNDO como un método de cálculo rápido para evaluar la energía LUMO de estos compuestos, de tal manera que pueda ser usado en el diseño de un algoritmo que clasifique rápidamente entre la actividad agonista o antagonista de nuevos compuestos sintetizados específicamente para esta subunidad.

\section{Agradecimientos}

Agradecimientos especiales al Dr. Leonardo René Lareo (Q.E.P.D.), que más que ser nuestro tutor, compartiendo con nosotros sus conocimientos, enseñándonos y guiándonos por el camino de la investigación, fue nuestro amigo brindándonos su cariño y llevándonos con sus enseñanzas de vida a lo que somos hoy.

\section{Financiación}

El artículo hace parte del proyecto de investigación \# 0290 financiado por la Vicerrectoría Académica de la Pontificia Universidad Javeriana.

\section{Conflicto de intereses}

Los autores no presentan ningún conflicto de intereses

\section{Referencias}

1. Petrovic M, Horák M, Sedlácek M, Vyklický L. Physiology and pathology of NMDA receptors. Prague Medical Report. 2005; 106 (2):113-136.
2. Kleckner NW, Dingledine R. Requirement for glycine in activation of NMDA-receptors expressed in Xenopus oocytes. Science. 1988; 241: 835.

3. MacDermott AB, Mayer ML, Westbrook GL, Smith SJ, Barker JL. NMDA-receptor activation increases cytoplasmic calcium concentration in cultured spinal cord neurons. Nature. 1986; 321: 519.

4. Nowak L, Bregestovski P, Ascher P, Herbet A, Prochiantz A. Magnesium gates glutamate-activated channels in mouse central neurons. Nature. 1984; 307: 462 .

5. Mayer ML, Vyklicky L, Clements J. Regulation of NMDA receptor desensitization in mouse hippocampal neurons by glycine. Nature. 1989; 338: 425.

6. Ishii T, Moriyoshi K, Sugihara H, Sakurada K, Kadotani H, Yokoi M, Akazawa C, Shigemoto R, Mizuno N, Masu M. Molecular characterization of the family of the N-methyl-d-aspartate receptor subunits. The Journal of Biological Chemistry. 1993; 2836.

7. Meguro H, Mori H, Araki K, Kushiya E, Kutsuwada T, Yamazaki M, Kumanish T, Arakawa M, Sakimura K, Mishina M. Functional characterization of a heteromeric NMDA receptor channel expressed from cloned cDNAs. Nature. 1992; 357: 70.

8. Cull-Candy SG, Leszkiewicz DN. Role of distinct NMDA receptor subtypes at central synapses. Science. 2004; 16:1-9.

9. Marvizon JC, Baudry M. Allosteric interactions and modulator requirement for NMDA receptor function. The European Journal of Pharmacology. 1994; 269: 165 .

10. Peters S, Koh J, Choi DW. Zinc selectively blocks the action of NMDA on cortical neurons. Science. 1987; 236: 589-593.

11. Kloog Y, Haring R, Sokolovsky M. Kinetic characterization of the phencyclidine-N-methyl-d-aspartate receptor interaction: evidence for a steric blockade of the channel. Biochemistry. 1988; 27 (3): 843-848.

12. Halliwell RF, Peters JA, Lambert JJ. The mechanism of action and pharmacological specificity of the anticonvulsant NMDA antagonist MK-801: a voltage clamp study on neuronal cells in culture. The British Journal of Pharmacology. 1989; 2: 480-494.

13. Brown DG, Urbanek RA, Bare TM, McLaren FM, Horchler CL, Murphy M, Steelman GB, Empfield JR, 
Forst JM, Herzog KJ, Xiao W, Dyroff MC, Lee ChiMing C, Trivedi S, Neilson KL, Keith RA. Synthesis of 7-chloro-2,3-dihydro-2-[1-(pyridinyl)alkyl]pyridazino[4,5-b]quinoline-1,4,10 $(5 \mathrm{H})$-triones as NMDA glycine-site antagonists. Bioorganic \& Medicinal Chemistry Letters. 2003; 13: 3553.

14. Salituro FG, Tomlinson RC, Baron BM, Demeter DA, Weintraub HJR, McDonald LA. Design, synthesis and molecular modeling of 3-acylamino-2-carboxyindole NMDA receptor glycine-site antagonists. The Journal of Biological Chemistry. 1991; 1: 455.

15. Smith ECR, McQuaid LA, Calligaro DO, O'Malley PJ. Structure-activity relationships of a series of glycine antagonists related to 5,7-dichlorokynurenic acid and 3-(2-carboxy-6-chloroindol-3-yl)acetic acid. Bioorganic \& Medicinal Chemistry Letters. 1993; 3: 81 .

16. Hays SJ, Boxer PA, Taylor CP, Vartanian MG, Robichaud LJ, Nielsen EØ, N-Sulfonyl derivatives of 6,7-dichloro 3,4-dihydro-3-oxo-quinoxalinecarboxylate as glycinesite NMDA and AMPA antagonists. Bioorganic \& Medicinal Chemistry Letters. 1993; 3:77.

17. Rowley M, Leeson P, Grimwood S, Marshall G, Saywell K. 5,6,7,8-Tetrahydroquinolones as antagonists at the glycine site of the NMDA receptor. Bioorganic \& Medicinal Chemistry Letters. 1995; 5: 2089.

18. Jackson PF, Davenport TW, Garcia L, McKinney JA, Melville MG, Harris GG, Chapdelaine MJ, Damewood JR, Pullan LM, Goldstein JM. Synthesis and biological activity of a series of 4-aryl substituted benz[b]azepines: antagonists at the strychnineinsensitive glycine site. Bioorganic \& Medicinal Chemistry Letters. 1995; 5: 3097.

19. Ghidini E, Delcanale M, Servadio V, Pietra C, Bergamaschi M, Villetti G, Redenti E, Ventura P, Merlini L. Synthesis of a new series of N-hydroxy, Nalkylamides of aminoacids as ligands of NMDA glycine site. The European Journal of Medicinal Chemistry. 1999; 34: 711.

20. Cugola A, Donati D, Guarneri M, Micheli F, Missio A, Pecunioso A, Reggiani A, Tarzia G, Zanirato V. Synthesis and biological evaluation of pyrido[2,3b]pyrazine and pyrido[2,3-b]pyrazine-N-oxide as selective glycine antagonists. Bioorganic \& Medicinal Chemistry Letters. 1996; 22: 2749.

21. Di Fabio R, Tranquillini E, Bertani B, Alvaro G, Micheli F, Sabbatini F, Pizzi MD, Pentassuglia
G, Pasquarello A, Messeri T, Donati D, Ratti E, Arban R, Dal Forno G, Reggiani A, Barnaby RJ. Enantiomerically pure tetrahydroquinoline derivatives as in vivo potent antagonists of the glycine binding site associated to the NMDA receptor. Bioorganic \& Medicinal Chemistry Letters. 2003; 3: 3863.

22. Colotta V, Catarzi D, Varano F, Calabri FR, Filacchioni G, Costagli C, Galli A. 3-Hydroxy-quinazoline-2,4dione as a useful scaffold to obtain selective Gly/ NMDA and AMPA receptor antagonists. Bioorganic \& Medicinal Chemistry Letters. 2004; 14 (9): 2345-2349.

23. Di Fabio R, Micheli F, Baraldi D, Bertani B, Conti N, Dal Forno G, Feriani A, Donati D, Marchioro C, Messeri T, Missio A, Pasquarello A, Pentassuglia G, Pizzi DA, Provera S, Quaglia AM, Sabbatini FM. Benzoazepine derivative as potent antagonists of the glycine binding site associated to the NMDA receptor. Farmaco Journal. 2003; 58 (9): 723-738.

24. Katayama S, Ae N, Kodo T, Masumoto S, Hourai S, Tamamura C, Tanaka H, Nagata R. Tricyclic indole-2carboxylic acids: highly in vivo active and selective antagonists for the glycine binding site of the NMDA receptor. The Journal of Medicinal Chemistry. 2002, 46 (5): 691-701.

25. Zhou ZL, Kher SM, Cai SX, Whittemore ER, Espitia SA, Hawkinson JE, Tran M, Woodward RM, Weber E, Keana JF. Synthesis and SAR of novel di- and trisubstituted 1,4-dihydroquinoxaline-2,3-diones related to licostinel (Acea 1021) as NMDA/glycine site antagonists. Bioorganic \& Medicinal Chemistry Letters. 2003; 11 (8): 1769-1780.

26. Yosa J, Blanco M, Acevedo O, Lareo LR. Molecular orbital differentiation of agonist and antagonist activity in the GlycineB-iGluR-NMDA receptor. The European Journal of Medicinal Chemistry. 2009; 44 (7): 2960-2966.

27. Shapiro SS, Wilk MB. An analysis of variance for normality (complete samples). Biometrika. 1965; 52:591-611.

28. Chakravarti, IM, Laha, RG, Roy J. Handbook of Methods of Applied Statistics, vol. I, John Wiley and Sons (1967): 392-394.

29. Pople JA, Santry DP, Segal GA. Approximate SelfConsistent Molecular Orbital Theory. I. Invariant Procedures. The Journal of Chemical Physics. 1965; 43:(S129-S135). 